\title{
KAWASAKI DISEASE WITH HEMOPHAGOCYTIC LYMPHOHISTIOCYTOSIS IN A CHILD WITH STXBP2 MONOALLELIC MUTATION
}

Sérgio Alves', Sofia Ferreira', Ana Carriço², Mafalda Santos ${ }^{3}$,Tereza Oliva ${ }^{4}$, Diana Moreira' I - Paediatrics department; 2 - Paediatrics Cardiology department; 3 - Paediatric Orthopaedics department; Centro HospitalarVila Nova de Gaia/ Espinho,Vila Nova de Gaia, Portugal 4 - Paediatric Oncology; IPO - Porto, Portugal

\section{INTRODUTION}

$\checkmark$ Secondary Hemophagocytic Lymphohistiocytosis (HLH) is a rare and potentially life-threatening complication of Kawasaki disease (KD).

$\checkmark$ This entity resembles primary $\mathrm{HLH}$, a group of immune dysregulation syndromes, inherited in an autosomal recessive fashion.

$\checkmark$ Genetic polymorphisms may play a central role in secondary HLH.

\section{CASE REPORT}

\section{4-year-old white boy, no past medical history}

\section{6-day history of fever, diffuse erythema, odynophagia and right foot pain.}

Physical examination - Cheilitis. Strawberry tongue (Figure I).

Conjunctival hyperaemia. Perineal rash with desquamation. Feet oedema.

\section{Initial blood work}

- Haemoglobin 9.4g/dL;WBC 9600/uL; Platelets 103.000/uL

- ESR $35 \mathrm{~mm} / \mathrm{I}^{\circ} \mathrm{h}$;Albumin $3,2 \mathrm{~g} / \mathrm{dL}$; triglycerides $162 \mathrm{mg} / \mathrm{dL}$

- TGO: $19 \mathrm{U} / \mathrm{L} ; \mathrm{TGP}: 17 \mathrm{U} / \mathrm{L} ; \mathrm{CRP} 2.9 \mathrm{mg} / \mathrm{dL}$

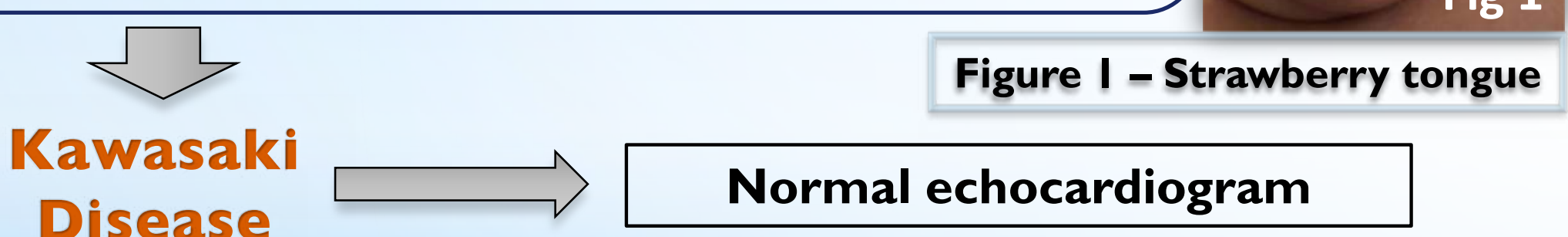

Intravenous immunoglobulin (IVIG) and acetylsalicylic acid.
(D7)

Right ankle swelling

Surgical bone drainage Cefuroxime iv (20 days)

MS Staphylococcus aureus Clin Clindamycin ( 10 weeks)

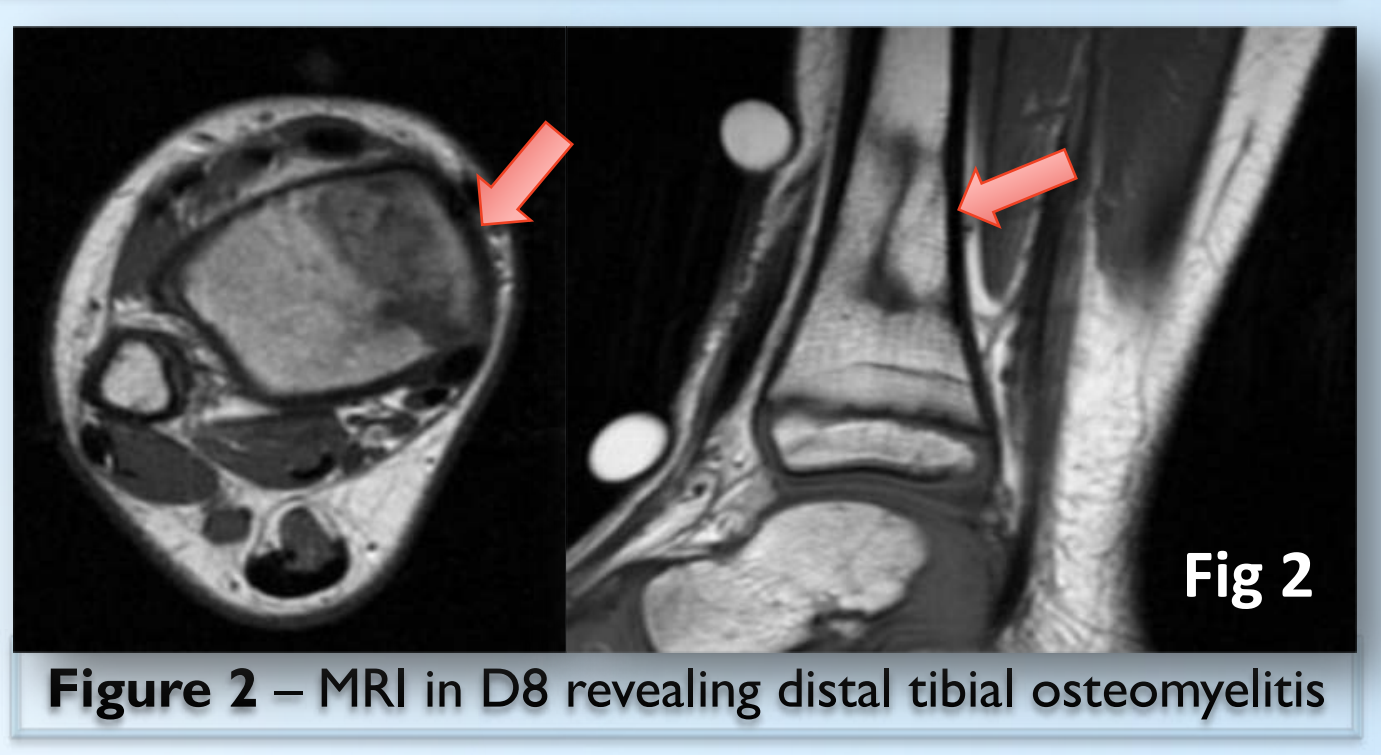

D20

Fever, exanthema and splenomegaly

$\mathrm{Hg} 7,8 \mathrm{~g} / \mathrm{dL} ; \mathrm{WBC} 2320 / \mathrm{uL}$ (neut 80/uL)

Platelets 135000/uL

ALT 22 I U/L; ferritin $36036 \mathrm{ng} / \mathrm{mL}$;

Fibrinogen $247 \mathrm{mg} / \mathrm{dL}$; SCD25 3346U/ml.

$$
\begin{aligned}
& \text { Hemophagocytic } \\
& \text { syndrome } \\
& \text { - Reference unit. } \\
& \text { - Bone marrow biopsy negative } \\
& \text { for hemophagocytosis }
\end{aligned}
$$

Conservative approach was assumed $\longrightarrow$ Gradual clinical and analytical normalization

9 months Bone marrow examinations - Hemophagocytic cells

- no other hemophagocytic criteria

Monoallelic after

- Regular outpatient vigilance

discharge STXBP2 mutation

\section{DISCUSSION}

$\checkmark$ Type 5 Familial HLH is caused by biallelic STXBP2 mutation. Heterozygosity may grant hemophagocytosis susceptibility, when triggered by conditions, such as KD or infections.

$\checkmark$ Optimal approach guidelines and specific HLH criteria for KD are necessary.

$\checkmark$ We highlight the importance of genetic study in these patients, in order to anticipate possible future recurrence.

I - García-Pavón S, Yamazaki-Nakashimada MA, Báez M, Borjas-Aguilar KL, Murata C. Kawasaki Disease Complicated With Macrophage Activation Syndrome.J Pediatr Hematol Oncol. 20I7;39(6):445-5I. 2 - Seo JY, Lee KO, Yoo KH, Sung KW, Koo HH, Kim SH, et al. Prevalence of type 5 familial hemophagocytic lymphohistiocytosis in Korea and novel mutations in STXBP2. Clin Genet. 2016;89(2):222-7.

3 - Wang W, Gong F, Zhu W, Fu S, Zhang Q. Macrophage activation syndrome in Kawasaki Disease: More common than we thought? Semin Arthritis Rheum. 2015;44(4):405-I0. 